Article

\title{
An Agent Based Model of Household Water Use
}

\section{Lilli Linkola $^{1, *}$, Clinton J. Andrews ${ }^{2}$ and Thorsten Schuetze ${ }^{3}$}

1 Sitra, PL 160, Helsinki 00181, Finland

2 Edward J. Bloustein School of Planning and Public Policy, Rutgers University, 33 Livingston Avenue, New Brunswick, NJ 08901, USA; E-Mail: cja1@rutgers.edu

3 Department of Architecture, Sungkyunkwan University, Suwon 440-746, Korea;

E-Mail: t.schuetze@skku.edu

* Author to whom correspondence should be addressed; E-Mail: lilli.linkola@gmail.com;

Tel. +358-294-618-310.

Received: 18 May 2013; in revised form: 5 July 2013 / Accepted: 5 July 2013/

Published: 17 July 2013

\begin{abstract}
Households consume a significant fraction of total potable water production. Strategies to improve the efficiency of water use tend to emphasize technological interventions to reduce or shift water demand. Behavioral water use reduction strategies can also play an important role, but a flexible framework for exploring the "what-ifs" has not been available. This paper introduces such a framework, presenting an agent-based model of household water-consuming behavior. The model simulates hourly water-using activities of household members within a rich technological and behavioral context, calibrated with appropriate data. Illustrative experiments compare the resulting water usage of U.S. and Dutch households and their associated water-using technologies, different household types (singles, families with children, and retired couples), different water metering regimes, and educational campaigns. All else equal, Dutch and metered households use less water. Retired households use more water because they are more often at home. Water-saving educational campaigns are effective for the part of the population that is receptive. Important interactions among these factors, both technological and behavioral, highlight the value of this framework for integrated analysis of the human-technologywater system.
\end{abstract}

Keywords: agent based modeling; behavioral factors; residential water use; buildings 


\section{Introduction}

Domestic water use constitutes a major part of urban water demand and is gaining importance regarding the sustainability of urban water supply. Households in the United States (U.S.) and The Netherlands (NL) use more than half of publicly supplied water [1,2]. The available water supply in urban areas, especially, is facing drastic declines in quantity and in quality and it is urgent to re-engineer the water services industry and better understand the demand side [3].

The total amount of drinking water used and wastewater discharged varies significantly across households. The measured standard deviations in mean indoor water consumption per capita and day are 161 L in Mayer and DeOreo [4] and 169.6 L in Heinrich [5]. Water consumption shows up as one of the most user sensitive building utilities, and total water consumption levels can be significantly influenced by changes in user practices and habits alone [6,7].

At home, we are surrounded by misaligned economic, technological and socio-cultural incentives that lead us to create wasteful water consumption patterns [3,8]. This problem of individual agency [9] functions as a starting point for the current research. Although water saving technology, such as the adoption of water saving fixtures, is an effective means to reduce domestic water consumption [10,11], the aim of the research is to understand, define and quantify how demographic, attitudinal and economic factors produces variance in household behavior, and thus influence water flows within buildings and in the overall water system.

Building information modeling tools aid system design, provide technical descriptions of the "hardware", and facilitate the efficient layout of water supply systems. The "software" characterizing individual end-user behavior and water use patterns typically remains implicit. Models assuming average occupant behavior do not adequately account for observed building water performance. In order to map the complex process of domestic water use, we require integrated social-psychological and technological models [12]. This paper introduces an agent based model (ABM) of domestic water use, which aims to address the dynamic behavior of dwellers (occupants) and simulate water consumption processes from the bottom up. Such a model has the potential to complement traditional technology oriented models of more efficient domestic water use, such as WiseWater $[13,14]$.

The model discussed in this paper generates realistic data on domestic water system consumption patterns at the individual and household level. The tool could be used to address issues, measures and incentives influencing the behavior of real users in empirically credible environments and to model the potential effects on domestic water use. The "hardware" included in the model consists of common water fixtures and appliances that enable domestic water use with different efficiencies. These are taps for washbasins in kitchens and bathrooms as well as for bathtubs, showers, washing machines, dishwashers and toilets. This study focuses on the quantity of drinking water consumed indoors and does not include uses for irrigation.

After the discussion of the model and exemplary modeling results this paper concludes with a methodical discussion of the investigated ABM and its utilization as a tool for studying domestic water systems as well as developing urban water conservation strategies and policies. Finally the paper makes suggestions for future research. 


\section{Materials and Methods}

ABMs are used to create a flexible abstraction of the real world. They consist of basic computer algorithm units, so-called agents. ABMs take agents as components of a larger entity and their interactions as central modeling focus points [15]. They are constructed to discover how system properties emerge from a bottom-up perspective. The key idea in agent-based thinking is that system behavior is not controlled by a single component, but that it is a result of the accumulated effect of decisions made by individual agents. In an ABM, the quantity of domestic water use is an aggregate outcome of occupants' individual water use decisions; adjusting the fixtures to a desired flow-rate while using them and enacting daily habits.

The choice of the modeling methodology was guided by the authors' wish to simulate autonomous water use decision makers, actual building occupants in a credible environment. Mathematical models with differential equations that represent certain changes of a certain quantity as a function of other quantities might be good in making predictions about the behavior of the system under different initial conditions, but their limitation is that they often capture only the average behavior of the system [16]. Experiments with the ABM allow users to quickly improve their intuition and understanding of the overall domestic water system [17].

Agent based modeling has been successfully used in simulating consumer behavior and technological lock-ins as a cellular automaton [18] and modeling possible trade-offs resulting from urban water system governance [17]. Typical ABMs includes four elements: environment, decision making, interactions, and adaptation. The modeling assumptions in the water use model are the following:

1. Water use fixtures and appliances are represented in the model environment;

2. Individual decision-making processes are represented with a combination of heuristic and stochastic rules;

3. Agents interact with their environment (controllability of fixtures and appliances and household level tasks);

4. Adaptive behavior is implemented through strategy selection.

The decision-making model adopted in this study is the one developed for a behaviorally-based building lighting model [19]. The decision-making model is a hybrid of two decision-making theories, the Theory of Planned Behavior (TPB) [20], which is used in the field of psychology, and the Belief-Desire-Intention (BDI) framework, a common tool for simulation modeling.

According to the TPB, human action is guided by three kinds of considerations: behavioral beliefs, normative beliefs and control beliefs [20]. In their respective aggregates behavioral beliefs produce a favorable or unfavorable attitude towards the behavior; normative beliefs result in perceived social pressure or subjective norms; and control beliefs give rise to perceived behavioral control. The BDI models seek to mimic the practical reasoning processes by which humans make the "right" decisions given the structure of their personal values and social norms [21]. Once a person (an occupant) forms an intention, e.g., to get clean, he reflects on all the options he perceives to achieve the desired outcome [19]. Again, the decision concerning which of the options to pursue (e.g., to take a bath or a shower) should depend on the agent's attitudes, desires, subjective norms and the locus of control (the extent to which one has control over the fixtures and appliances, thus over one's water usage). 
A set of rules to represent the decision-making processes of occupants in the ABM as they interact with water system components and one another were based on the relevant literature.

Statistical data about the domestic water use for the United States of America (USA) [4] and The Netherlands [22] was used to validate the model and to give an overview about the average domestic water consumption, in total and for specific purposes.

The ABM for domestic water use discussed in this paper was developed within a free open-source modeling environment called Netlogo [23]. The selected program was designed for developing simple multi-agent models and enables rapid model developments. Furthermore the open-source software enables researchers to reproduce the presented method and results.

\section{Model Description}

\subsection{Elements and Structure of Domestic Water Use System}

The system to be observed is the domestic indoor water consumption system, which includes three conceptual levels: (1) indoor level; (2) building level; and (3) outside world level, as illustrated in Figure 1. The indoor level includes the direct determinants of domestic water consumption - the technological infrastructure in place together with the occupants' behavior. The building constrains the system and directs occupants' behavioral choices. The outside world level defines the overall framework in which water consumption takes place.

Figure 1. The three main levels influencing the water consumption of a building: (1) Indoors are situated the core elements of the domestic water system, water related technologies and activities inside the building; (2) The building type defines the frame, the building itself as well as its equipment and use; (3) The World includes the context of water use. These are all factors affecting the water technologies and water use behavior in a specific environment and society.

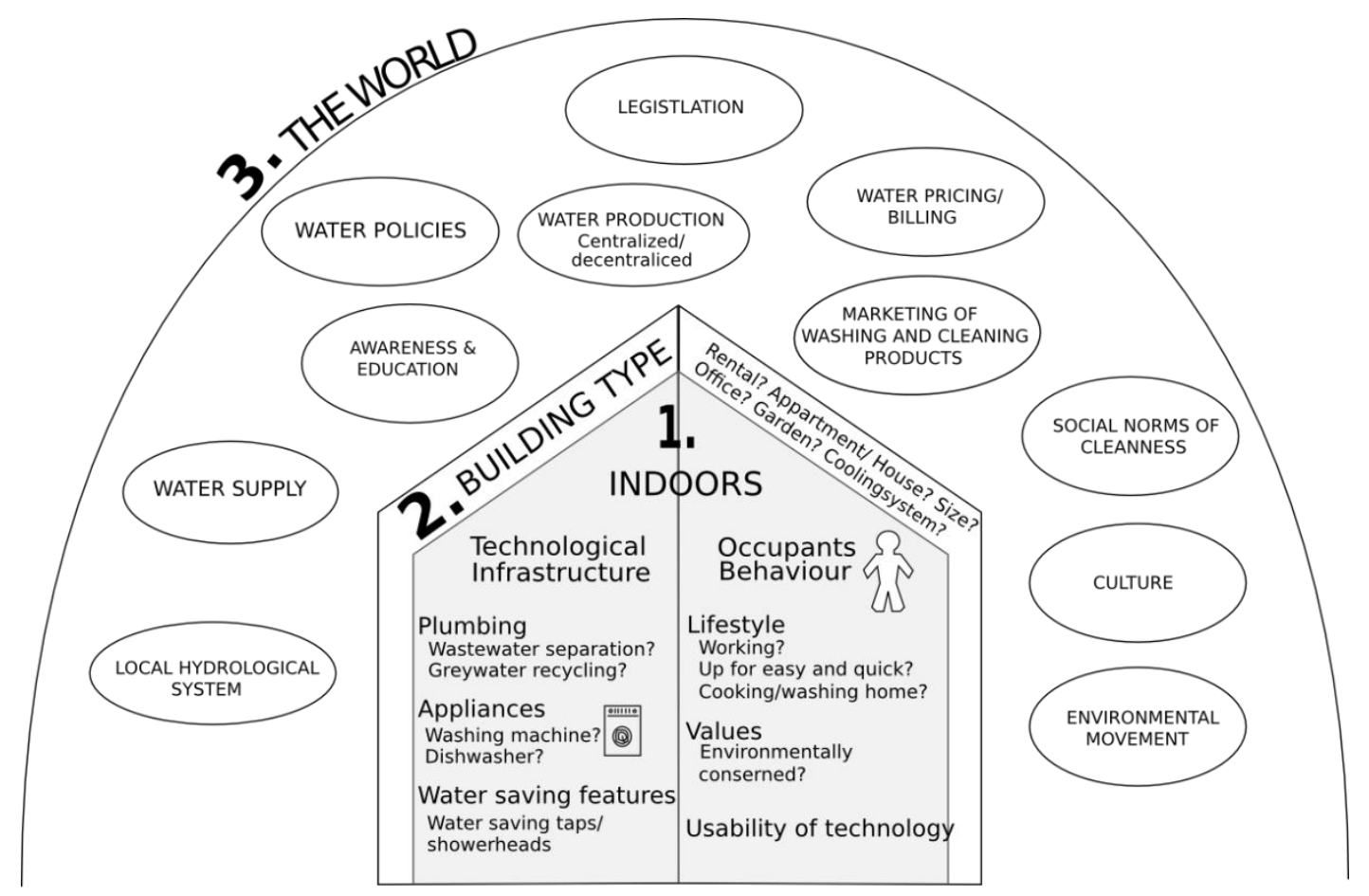




\subsection{Entities, States, Variables and Scales}

As the building water consumption system is translated to an ABM, the building water infrastructure and the occupants inside the building (Figure 1) become the physical environment and the agents respectively. The term "building occupant" refers to the real world entity of humans in residential apartments and the notion 'agent' refers to the modeling interpretation of this real world entity.

Individual agents form households, which are the sampling unit of the simulations, the system to be observed. The designed ABM for domestic water use simulates the water usage of one household at a time.

Agents have daily habits, which describe the frequency with which they engage in certain water consuming practices. The agents are assigned with the following water use practices in the model set-up: cleaning the body (personal hygiene), cooking and drinking, doing laundry, using the toilet and washing dishes.

The probable frequencies of specific practices, the "daily habits", are not directly translated into actual water usage in the model as the agents also follow the decision-making process each time the change in agent state triggers agents to think about their next actions. The habits and the heuristics for decision making and behavior are the rules of agent behavior in the model.

Behavior, in the context of this research, is defined as the aggregated response of an agent to incentives and inputs, whether external or internal and conscious or subconscious. The agents have a set of behavioral variables (listed in Table 1), which define the agent state. Agent state and rules are affected by agent parameters (age and employment status) and situational factors; the household parameters and variables (Figure 2).

Table 1. A list of the model behavioral variables.

\begin{tabular}{|c|c|c|}
\hline Variable & Value & Description \\
\hline \multicolumn{3}{|c|}{ Global Variables } \\
\hline Laundry stock & $\geq 0 \mathrm{~kg}$ & $\begin{array}{l}\text { State variable. Describes how much laundry is left to wash. } \\
\mathrm{kg} \text { of laundry/day }=1.3 \text {-agent intention to save water. }\end{array}$ \\
\hline Dish stock & $\geq 0$ plate sets & State variable. Describes how many dishes are left to wash. \\
\hline Hours & $\geq 0 \mathrm{~h}$ & \\
\hline Days & $\geq 1$ day & \\
\hline Weeks & $\geq 1$ week & \\
\hline
\end{tabular}

Agent Variables

At-home? true/false

State variable. Describes if agent is at home or not. It

$\begin{array}{ll}\text { Working? } & \begin{array}{l}\text { true/false (GUI) } \\ \text { sustenance/eliminate/bodyCare/ } \\ \text { cleanClothes/dishWash }\end{array}\end{array}$
is assumed that working agents spend twice as little time at home as non-working agents. Agent employment status.

Agent's current state.

State variable. Describes how the agent is thinking of Plan doing the current action. The plan is a random draw from the bcstrategy (body care strategy), wcstrategy and eatstrategy lists. age group and employment status. Children tend to have more baths, employed agents more frequent showers. 
Table 1. Cont.

\begin{tabular}{|c|c|c|}
\hline Variable & Value & Description \\
\hline \multicolumn{3}{|c|}{ Agent Variables } \\
\hline WC strategy & NA/excrete/urinate & The proportional need to excrete and urinate is $1: 4$. \\
\hline Eat strategy & $\begin{array}{l}\mathrm{NA} / \mathrm{drink} / \mathrm{cook} / \text { eat commercial } \\
\text { prepared food at home/eat } \\
\text { elsewhere }\end{array}$ & $\begin{array}{l}\text { It is assumed that average agent has three meals and } \\
\text { four drinks per day ( } 7 \text { events). }\end{array}$ \\
\hline IntentionToS & & See function (1). \\
\hline \multicolumn{3}{|c|}{ Patch variables } \\
\hline Water liters & $\mathrm{L}$ & $\begin{array}{l}\text { Liters of water consumed through each fixture and } \\
\text { appliance. }\end{array}$ \\
\hline Times use & integer & Frequency of using each fixture and appliance. \\
\hline
\end{tabular}

Figure 2. System structure: The units of enquiry in the model are individual agents. Agents acquire information from the environment which affects agent state. Agents behave according to rules. Agents form households, the behavioral system to be observed. The household state is the result of the agent actions; we call it the aggregate state. Agent actions are triggered by their habits.

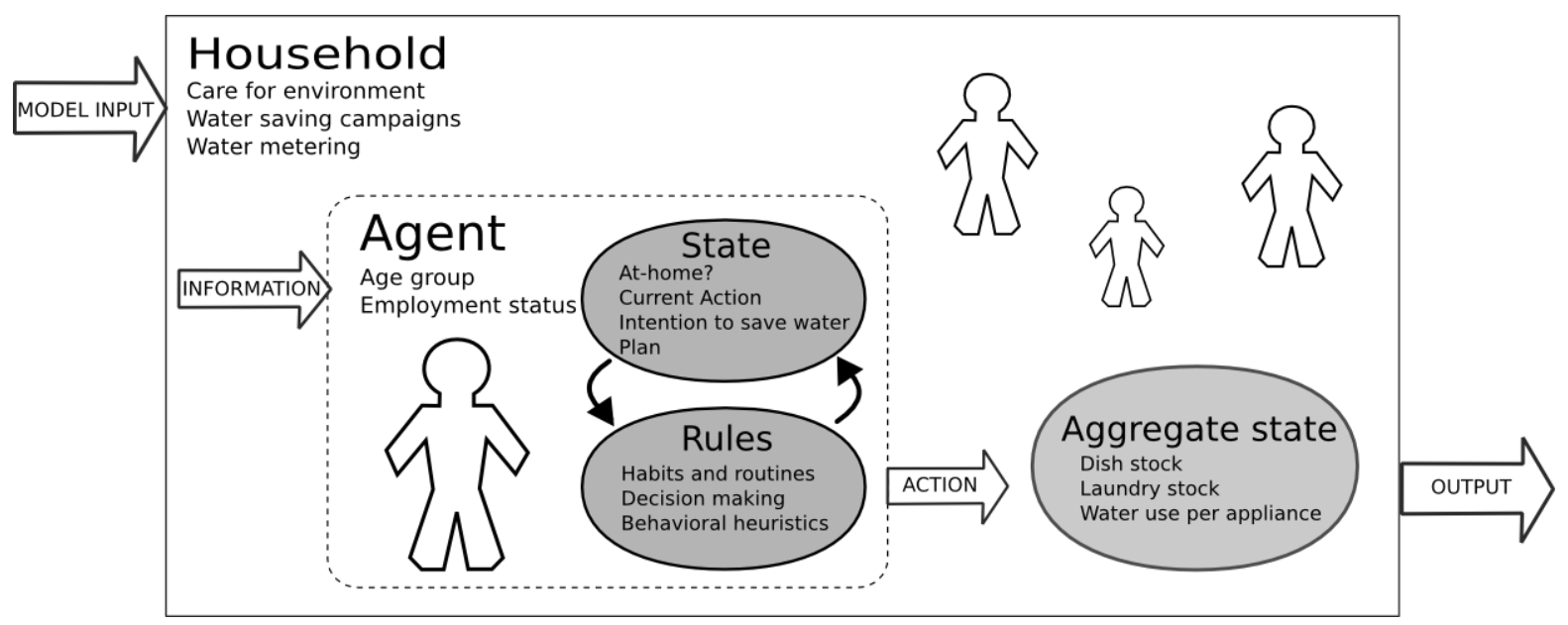

Each agent is initialized to be employed or unemployed based on whether the occupant is working or studying full time outside home or not. The agent belongs to a certain age group: a child (person under 12 years old), an adult (a person aged 12-63) or a senior (person over 63).

Age constrains the behavior of agents as children do not launder or wash dishes. The agent's employment status affects the hours the agent spends at home. Age and employment status are also important determinants of agent showering and bathing habits [24].

Other factors affecting the agent decision-making process are water saving campaigns, water metering and household values and resulting norms of acceptable water use behavior. The intention of individual agents to save water in the model is a function of these factors, which can be expressed as follows:

$$
\text { IntentionToSaveWater }=a+b+f
$$

where, $a=$ care for environment $/ 10$ if water saving campaigns $=$ true otherwise $a=0, b=0.4$ if water meter $=$ true otherwise $b=0$ and $f=0.1$ is a constant to avoid the value zero as equation outcome. 
According to the Schwartz value theory [25], "universalism" and "benevolence" result in self-transcendent behavior when they occur as dominant personality traits. It is assumed within the model that environmentally significant values and that the tendency to self-transcendent behavior, the level to which agents manifest care for environment in their daily habits are shared with the individuals within one household. The underlying logic is that each household would have its own norms of acceptable water use behavior.

Studies suggest that the mere introduction of metering, regardless of the pricing structure used, results in a reduction in water use of anywhere between $7 \%$ and $35 \%$ [26,27]. The impact of metering is difficult to separate from other measures which are often introduced simultaneously. Information campaigns seem a crucial addition in building up awareness over how to reduce water consumption and why it is important to do so [27].

The agent and household characteristics and the configuration of the model environment depend always on the model set-up, which can be defined in the model user interface. The model set-up parameters are listed in Table 2 with the exception of the parameters describing the technological configuration. The behavioral variables in the model are summarized in Table 1.

Table 2. A list of model set-up parameters.

\begin{tabular}{|c|c|c|}
\hline Variable & Value & Description \\
\hline \multicolumn{3}{|l|}{ Household } \\
\hline No. agents & $\mathrm{x}(\mathrm{GUI})$ & Number of household members \\
\hline Household type & & family with kids/family without kids/co-housing/living alone \\
\hline No. agents under 12 & $\mathrm{x}(\mathrm{GUI})$ & Number of children \\
\hline No. agents over 63 & $\mathrm{x}(\mathrm{GUI})$ & Number of seniors \\
\hline $\begin{array}{l}\text { No. agents working/ } \\
\text { studying outside home }\end{array}$ & $\mathrm{x}(\mathrm{GUI})$ & $\begin{array}{l}\text { Number of hours agent spends at home. It is assumed that } \\
\text { an agent studying/working spends eight hours less at home } \\
\text { than others. }\end{array}$ \\
\hline Care for environment & $0.0-5$ & $\begin{array}{l}\text { Care for environment by the agent, where } 0=\text { no care and } \\
5=\text { maximum care. }\end{array}$ \\
\hline \multicolumn{3}{|l|}{ Environment } \\
\hline Water meter & true/false & True if household water is metered, otherwise false \\
\hline Water saving campaigns & true/false & True if the household is exposed to water saving campaigns. \\
\hline \multicolumn{3}{|l|}{ Agent habits } \\
\hline Sustenance & 0.4375 times $/ \mathrm{h}$ & Frequency an agent eats/drinks, based on Wilkes [24]. \\
\hline Eliminate & 0.5 times $/ \mathrm{h}$ & It is assumed that average agent has 8 toilet visits per day. \\
\hline Body care kids & 0.0794 times $/ \mathrm{h}$ & Frequency agents under 12 clean body, based on Wilkes [24]. \\
\hline Body care adults & 0.0856 times $/ \mathrm{h}$ & Frequency agents clean body, based on Wilkes [24]. \\
\hline Body care seniors & 0.0637 times $/ \mathrm{h}$ & Frequency agents over 64 clean body, based on Wilkes [24]. \\
\hline Clothes wash & $1.3 \mathrm{~kg} /$ day & $\begin{array}{l}\text { Amount of laundry an agent generates per day, estimation } \\
\text { by author. }\end{array}$ \\
\hline
\end{tabular}

\subsection{Overview of Processes and Specific Timing}

The chronological order of the processes and agent actions at each time step of the model (one model tick) are represented in Figure 3. The time step in the model is hourly (hour = one model tick) and the model keeps track of days and weeks. First, the model updates its environment, the water 
system's technological configuration. Next, the program calls all agents to execute their water use habits in the same order as they are represented in the grey box in Figure 3. If an agent does not have an acute need, such as the need to clean their body, the agent skips the process and continues straight to examine its other needs, in this case the need for clean clothes. If the agent identifies a need, such as need to clean clothes, the agent continues to follow the decision-making process that guides the agent's choice of actions illustrated in Figure 4.

For each action there are behavioral options, plans from which the agent can choose. There are, for example, five different options available when an agent decides in the ABM program to clean his body: (1) to do nothing; (2) to take a shower; (3) to wash his face under faucet; (4) to get substitutes (which means that he did personal hygiene outside and not at home); and (5) to take a bath. According to the frequency with which the agent performs each plan, he/she forms strategies for that particular practice. For example, an agent can have a strategy to take four showers and one bath per week in order to clean his/her body.

The frequency in which the agent cleans the body, eats and drinks, and eliminates is stochastic. An agent's perception of its needs (derived from habits) and deliberation to do something is based on a random trial. The model chooses a random number between 0 and 1 , and if the number is greater than the agent's probability of doing whatever it deliberates to do the agent chooses to engage to the action (deliberation).

Figure 3. Agent action and general model processes scheduling during one time step of the model, which is one model tick. Agent actions and their order are listed in the grey box.

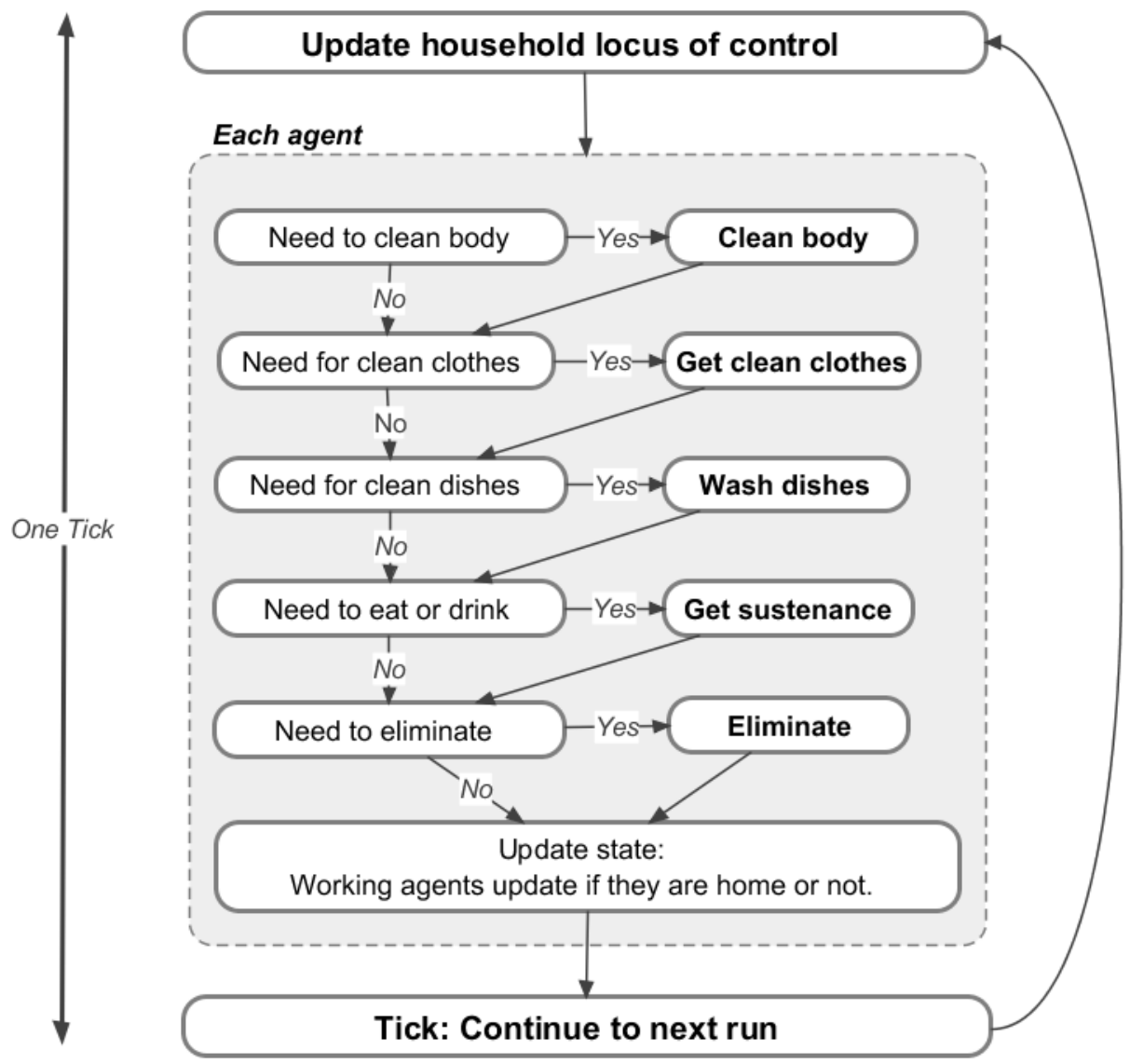


Figure 4. Graphical representation of the agent decision-making process in the model to execute each of the water use habits: clean body, get clean clothes, wash dishes, get sustenance and eliminate. An agent perceives its needs and desires, plans which actions to take, checks the household locus of control and intention to save water, and finally decides and executes.

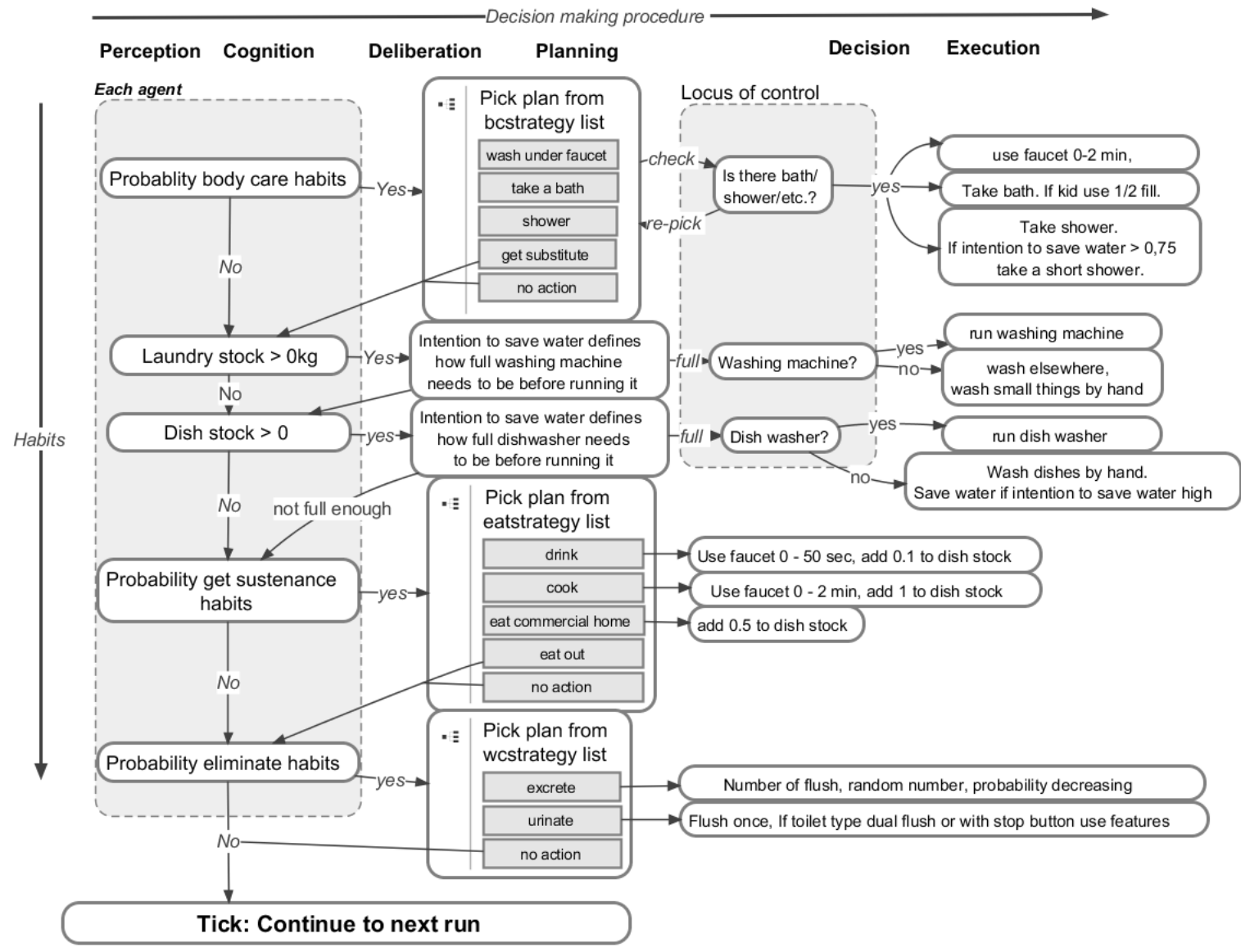

\section{Results and Discussion}

\subsection{Description of the Experiments}

The information on average water use technologies (including household appliances, fixtures and user interfaces) in place at home in Mayer and DeOreo [4] and Foekema et al. [22] was used to design two different pre-set configurations in the model environment: "U.S. apartment" and "NL apartment". Based on research findings and common practice in both countries, all households in "NL apartments" were set to be metered whereas the households in "U.S. apartments" were not.

The simulation experiments were performed in these two environmental set-ups and with three agent configuration scenarios:

1. single - a person living alone, having a busy life style, i.e., working fulltime outside home,

2. couple - a retired couple, spending most of their time at home, and

3. family - a family with two children, two of the members of the family working or going to school full-time outside home.

To accommodate path dependencies that derive from the many stochastic variables within the model, we show results for 100 repetitions for each household parameter settings (except for U.S. single 
300 repetitions). All together we had 1200 repetitions of each scenario in both of the environments, that is, 12 households with different levels of care for environment worth of data (except the case of U.S. single 3600 repetitions, $12 \times 300$ ). Box-and-whisker plots provide a visual summary of the results. The line in the middle of each box is the median, the box itself covers two quartiles (50\% of the variation), and the whiskers cover the remaining two quartiles (50\% excepting outliers).

To focus the research findings, the following three different hypotheses were formed.

Hypothesis 1 is that a busy lifestyle results in less water use in the kitchen and toilet. Two major facts from the literature support this hypothesis. There is less water used in toilets on weekdays compared to weekends [22]. A person living alone cooks fewer meals per day at home than people living in a household with two or more persons [28]. A study made by Kant and Graubard [29] also suggests that persons with high incomes and higher education levels tend to have more commercially prepared meals or eat more often outside.

Hypothesis 2 is that water conscious behavior does not emerge from environmental awareness and valuation alone but is also strongly encouraged by economic factors and an increase in occupant awareness by means of water metering and water saving campaigns [10,27,30].

Hypothesis 3 is that showering and bathing practices are mostly related to specific working status and underlying social norms. Findings in literature indicate that showering is more frequent amongst working aged people (in the age from 15 to 63 ) [4,22,24]. People who work or study outside the home follow the social norms present at their work place. In an environment where most people are freshly showered and clean, new employees tend to follow this trend and adopt frequent showering habits.

The three different occupant scenarios were tested against the hypotheses. All experimental set-ups were run for a period of one year. The observed variables were the total and per fixture water consumption and the frequency of usage of every fixture and appliance at the household level.

\subsection{Model Validation and Sensitivity Analyses}

The total average domestic water consumption in the USA is measured at $226.4 \mathrm{~L}$ per capita and day [4] and 127.5 L per capita and day in The Netherlands [22]. The aggregated outcomes of the three household scenarios in both environmental set-ups are somewhat in line with these measured averages, households having daily water consumption of $151.0 \mathrm{~L}$ and $237.7 \mathrm{~L}$ respectively. A more detailed comparison of the measured and modeled water end use averages is listed in Table 3.

More details on the sensitivity of the model experiments and results explained in the next section.

Table 3. Measured and modeled total average water consumption per capita and day in the USA and The Netherlands, in total and assigned specific purposes (1, [22] and 2, [4]).

\begin{tabular}{lcccc}
\hline Domestic Water Consumption (L/cap./day) & NL (1) & Model NL & USA (2) & Model USA \\
\hline Cooking \& Drinking (Kitchen Tap, USA all taps) & 8.8 & 20.2 & 41.3 & 15.5 \\
Washing (NL Kitchen Tap \& Dishwasher, rest dishwasher only) & 6.8 & 2.0 & 3.8 & 3.3 \\
Bathing (Bath \& Shower) & 52.3 & 96.0 & 48.4 & 101.1 \\
Laundry (Washing Machine) & 17.2 & 7 & 56.8 & 43.5 \\
Toilet Flushing (Toilet) & 37.1 & 24.9 & 70 & 73.3 \\
Other Indoor Usage (Mostly Tap) & 5.3 & 0.9 & 6.1 & 0.27 \\
\hline Total & 127.5 & 151.0 & 226.4 & 237.7 \\
\hline
\end{tabular}




\subsection{Differences in Water Use per Household Type}

The ABM results show significant variations in total water use levels and fixture specific water use levels between individual agents and household types.

The experiments revealed a pattern of a retired couple having the highest per agent water consumption level in both of the model environmental set-ups (Figure 5). The difference between the water consumption per agent of the single person and the family with two children is relatively small.

The distribution of simulation outcomes regarding water use per fixture, illustrated with box-plots in Figure 6, indicates that the range of probable total water consumed outcomes is the largest for showers and washing machines within these simulation runs.

The per-agent fixture usage varies substantially between the three different household types (single, couple and family in Figure 7), washing machine use being an exception. The agent living alone is the one who showers most frequently. The retired couple scores the highest in what amounts to frequency of using toilet and taps. The bath, on the other hand, is most utilized in families with children.

Dishwasher use is less apparently related to household type. In general there is quite some variance in dishwashing habits judging by the shape of the boxes in Figure 7 . They represent the middle 50\% of the observations of kitchen tap usage and dishwasher usage. This link between kitchen tap and dishwasher usage is rather natural as an agent may choose to wash dishes by hand or in a dishwasher. In addition, the more an agent cooks at home the more the agent generates dishes.

Figure 5. Distribution of water usage per agent per day by household type and country. The middle box is the $50 \%$ percentile, the bottom and top of the box are the $25 \%$ and $75 \%$ per-centiles of observations. Each boxplots represents 1200 model runs and 12 households worth of data, except for U.S. single which represents 12 households worth of data but has three times more 3600 model runs.

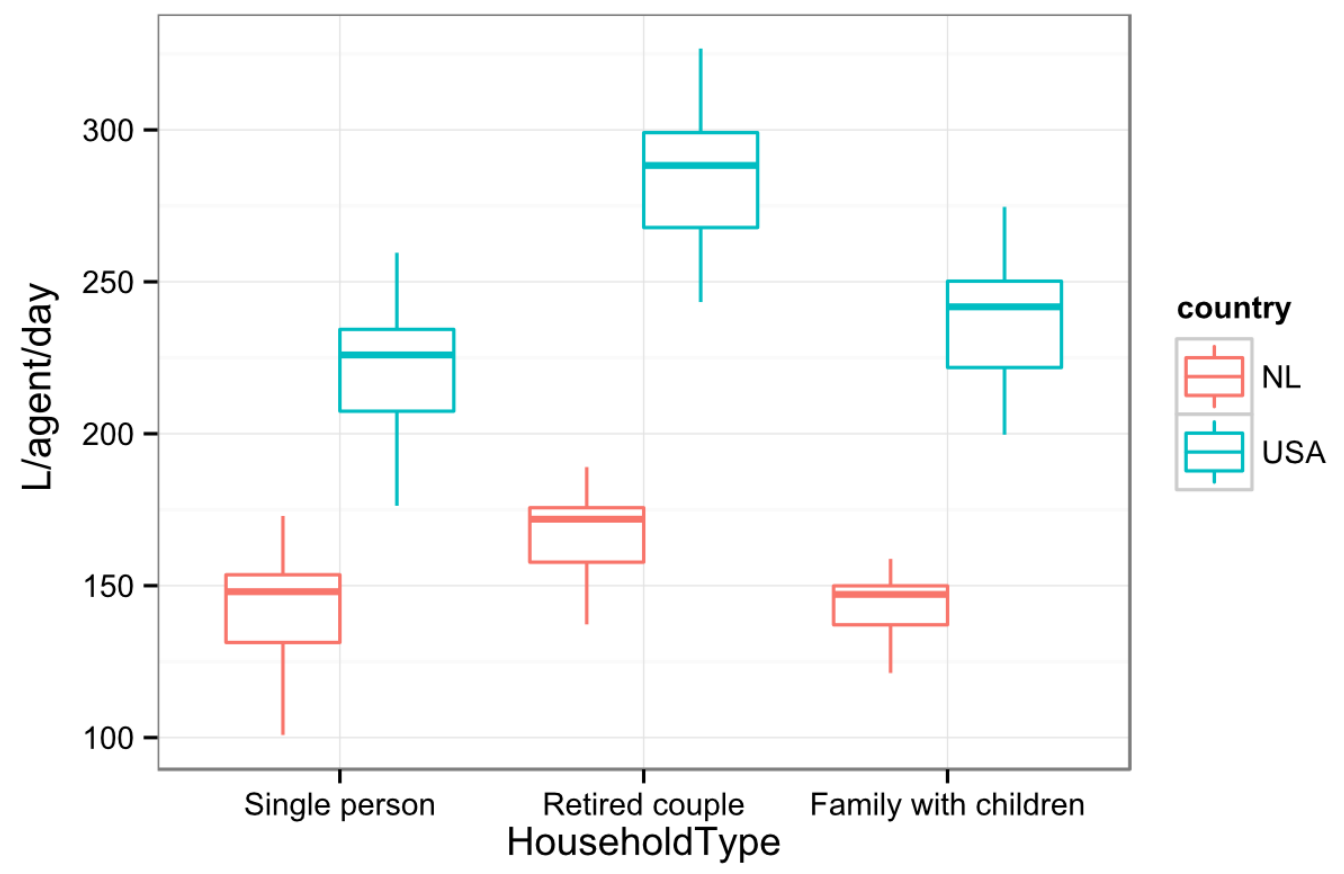


Figure 6. Distribution of total water usage in $\mathrm{m}^{3}$ per year per water fixture and per household type.

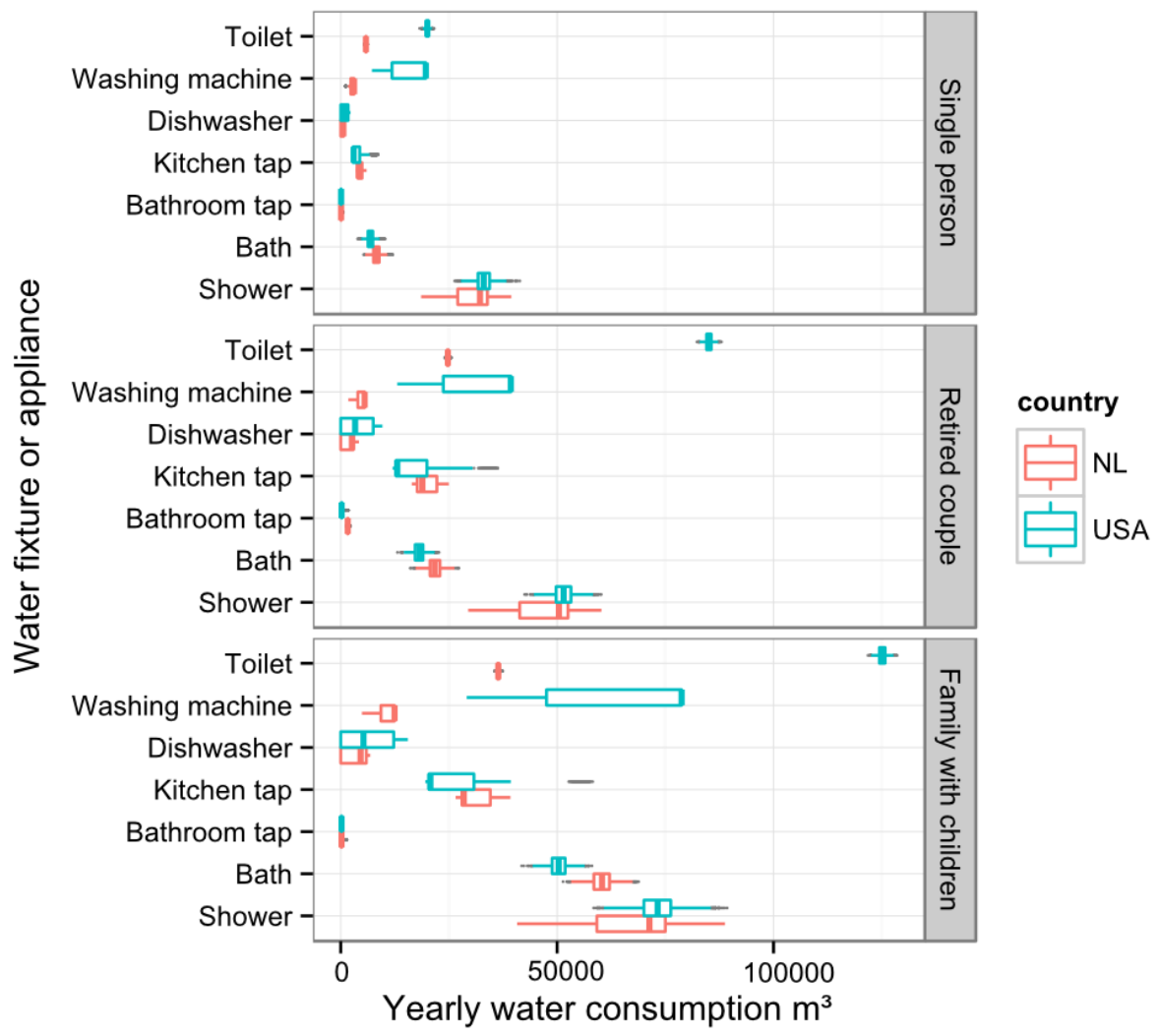

Figure 7. Distribution of fixture and appliance use frequency per year per water fixture and per household type. The differences in frequencies of use are the consequence of differences in agent behavior.

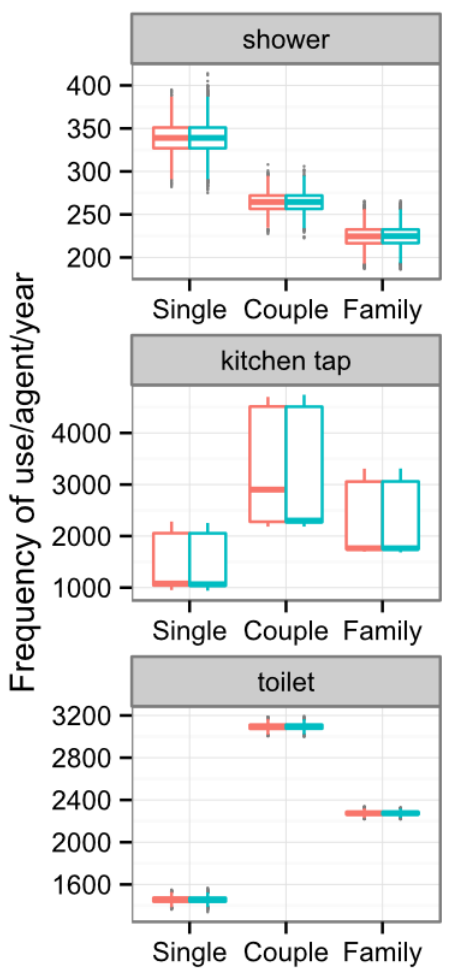

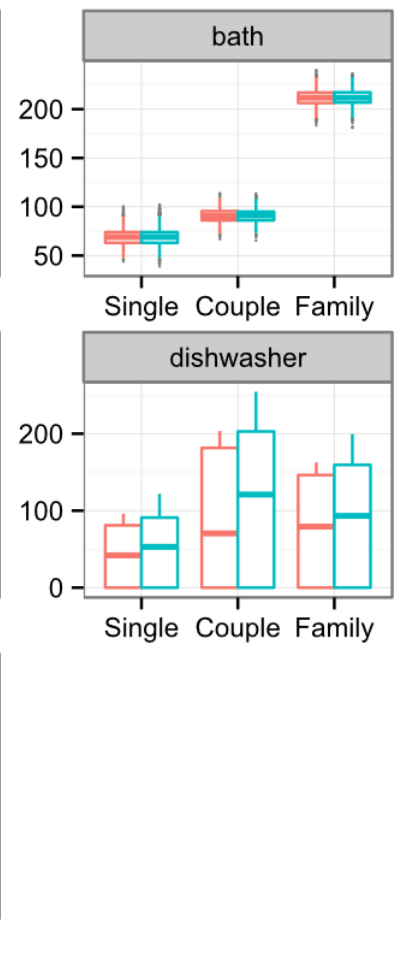

Household type 


\subsection{Contribution of Selected Behavioral Factors to Different Water Use Levels}

\subsubsection{Daily Schedules: Time Used at Home}

The difference of mean water use in the kitchen, including tap water use and dishwasher use, between working and retired agents living in a "U.S. home" is $16.5 \mathrm{~L} /$ agent/day, the working agent consuming $13.5 \mathrm{~L} /$ day and the retired agent $30 \mathrm{~L} /$ day. This result suggests that the agent working full time outside the home consumes over $50 \%$ less water in the kitchen. The toilet water use patterns are similar. The retired agent consumes 116.5 L/day in the toilet in the U.S. home whereas the working agent consumes only $54.8 \mathrm{~L} /$ day. Again, the water consumption of the working agent is over $50 \%$ less than that of the retired agent.

\subsubsection{Agent Incentives to Save Water}

Figure 8 shows how changes in parameters, namely the agent value for care for environment and the two external factors, water metering and water saving campaigns, result in different mean water consumption levels per day for a single.

Figure 8. A density plot of a one-person household in USA home mean total water consumption per day and how it is affected by water metering, ongoing water saving campaigns and the household level of care for the environment. Parameter sweeps are done by following model parameters: CareForEnvironment, WaterMeter and WaterSavingCampaigns. The agent care for environment is the lowest when it is 0 and highest when it is 5 . The number of samples for each specific parameter setting is $\mathrm{N}=900$.

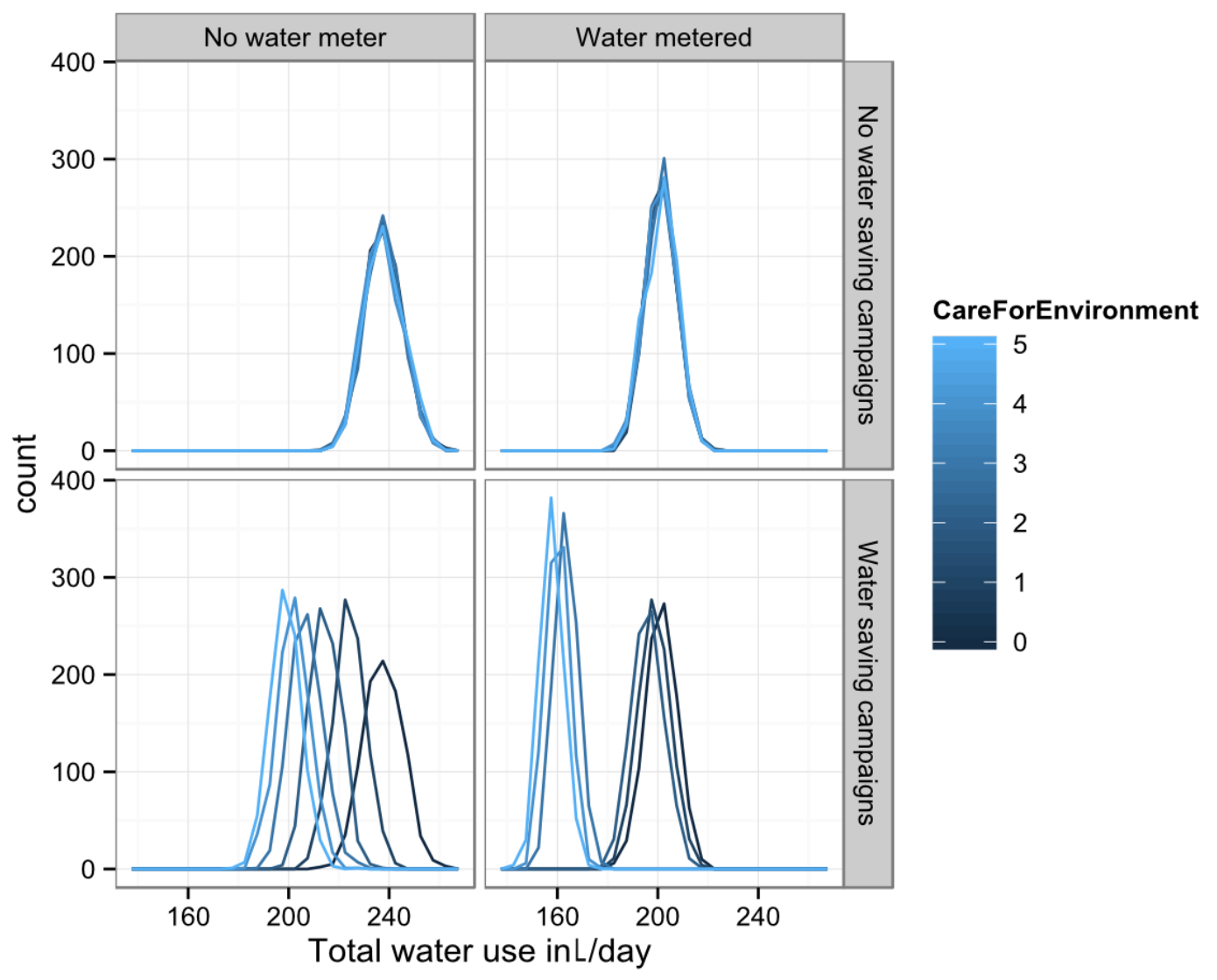


In situations where domestic water use is not metered and there is no systematic attempt to raise awareness among the water consumers over the importance to save water (the top left corner of Figure 8), the mean water has very little variance despite the different value of CareForEnvironment the occupants possess; the difference of means is statistically insignificant and the mean of all samples (5400) is $223 \mathrm{~L} /$ agent/day.

The introduction of water meters should result in an abrupt decrease in total water usage. This seems to be true in the situation presented in the upper right corner of Figure 8. Here again, the difference of means is statistically insignificant, however the mean of samples (5400) is considerably lower than in the previous case (no water meter), namely $200 \mathrm{~L} /$ cap/day.

More variation between the sampled different occupant types can be observed in the plot in the lower left-hand part of Figure 8. The occupant care for environment has impact on the simulated water consumption only if the occupant has awareness of the environmental impact of excessive water consumption. The model outcomes suggest that the agents that have a high level of CareForEnvironment consume on average 17\% less water (difference of means is $38 \mathrm{~L}$ ).

The plot in the lower right corner of Figure 8 demonstrates the agent total water usage in the situation where both external factors, water metering and water saving campaigns, are present. The mean water consumption in this category is $166 \mathrm{~L} /$ cap/day, $21 \mathrm{~L}$ less than in the latter case of campaigns but no metering. Again, agent environmental awareness affects water consumption.

The difference of means between all sampled levels of CareForEnvironment is statistically significant (based on Welch's two sample $t$-test in $\mathrm{R}$ ). The sample means are grouped in two value areas: around $190 \mathrm{~L} /$ day and $155 \mathrm{~L} /$ day. Around $185 \mathrm{~L}$ /day seems to be a water consumption level which the "busy" agent living alone rarely reaches unless it changes its habits more radically.

The sudden jump in the mean consumption level is caused by the agent's change in showering habits. The agent has two options for average showering durations, short and long (normal) shower. The assumed showering durations are based on Wilkes et al. [24]. The different showering durations can be clearly distinguished in Figure 8. Agents have a threshold value of IntentionToSaveWater $=0.75$ over which agents start to take shorter showers.

An intriguing detail in the plot is that the incremental reductions in agent total water use level becomes smaller and smaller as agent level of CareForEnvironment increases. There is very little difference in total per day water use level between agents that have the level 4 or 5 of CareForEnvironment.

\subsubsection{Social Norm of Cleanness}

The agents form showering and bathing habits based on their employment status and age. The showering habits change very little during the model run as there is no change introduced to environmental parameters. Thus, the model results related to agent bathing habits and total water use level are consistent with the expected outcomes: the household with a person living alone and working has the highest per agent frequency of showering and water consumption level in showering and bathing. See Figure 9 for comparisons of the relation between water consumption level and frequency of showering and bathing of all three household types. 
Figure 9. A scatter plot of agent bathing frequency (shower or bath) and water consumption in L/agent/day in these activities. The agents of different household types are distinguished with colors. Each single point represents the outcome of one model run. There are 21,000 observations for both family and couple and 64,800 observations for single.

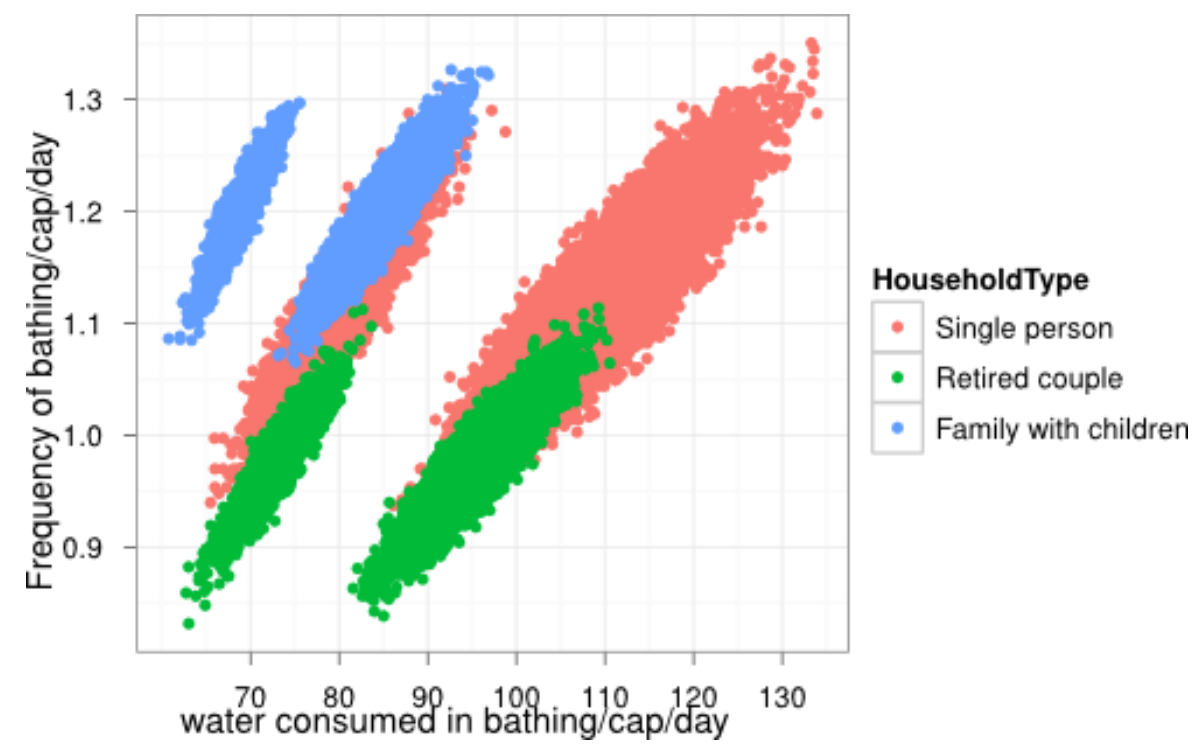

\subsection{Discussion}

The simulations show that the model 'works', meaning that the household water use levels are somewhat realistic and in line with the water end use data used as a base of the model. With simple behavioral rules we were able to recreate important variance in agent and household water use levels. A working agent who spends $50 \%$ less time at home results in $50 \%$ less water use in the kitchen and toilet and an agent who consciously makes an effort saving water uses $70 \mathrm{~L}$ less water than the agent who does not make this effort. The experiments demonstrated how the selected intentional behavioral incentives of water metering and water saving campaigns, and unintentional incentives including social norms of cleanness and time might translate into differences in water end use.

Based on the model experiment, agents seem to have threshold values of minimum water consumption level rooted in current habits and technologies. Water saving campaigns do not seem to add much more savings on top of water metering except for agents that were willing to compromise their showering enjoyment and choose to take shorter showers. Similarly, the smaller the current water use level, the smaller the further reductions are in water consumption that can be achieved with comfortable changes in agent behavior.

The relation between the time an agent spends at home and its water use levels in the kitchen and the toilet seems linear. It is difficult to judge how realistic these model outcomes are as there is hardly any empirical data on the correlation of the number of hours occupants spend at home with household water use levels.

The greatest contributor to water usage seems to be showering and bathing across all experiments. Compared to the data in Mayer and DeOreo [4], Foekema et al. [22] the water consumed in showering and bathing seems over estimated. Yet we chose not to change the occupants' showering frequencies, because they were based on recommended values [24]. The frequency used in Blokker et al. [31] is 
smaller and additionally the stochastic process is based on Bi-nominal distribution. For showering duration the agents were able to choose a gamma distributed random value. As the flow rate of common showers is controllable by its users, it might have led to a more realistic result if the shower flow rate would not have been by default the maximum one but equally a logically distributed value.

Technology restricts occupants' possibilities of performing water efficient behavior [32]. User satisfaction in most cases is derived from the services the activity of using water mediates, not from the water itself. The model can help policy makers to determine achievable water reductions within any existing situation and choose the most effective measures to reduce water consumption.

The designers of fixtures and appliances may use the model to define a range of use scenarios, minimum and maximum water demand and the characteristics of the distribution (tails and slopes) of the total water use. These factors may prove to be useful in optimizing the functionality of fixtures and appliances so that the water consumption would be minimized or the cost effectiveness of design choices maximized.

The methodological choice to use ABM to model human behavior, in the light of this research, seems still to be appropriate. The model yields many applications and is easy to extend to different purposes by adding new metrics.

Very sophisticated modeling of human decision making is laborious and we are limited by the empirical data on occupant behavior to construct behavioral heuristics and validate the model. For this reason the experiments with the model described in this paper resemble to some extent stochastic simulations. Modeling approaches such as the Monte Carlo method might accommodate better the uncertainties due to human decision making and be sufficient to model possible use scenarios.

NetLogo is not per se designed for modeling sophisticated decision-making processes and there are only a few applications of intelligent human agents (sometimes called BDI agents) in NetLogo models. This is a disadvantage, as implementation of an ABM is not straightforward. Behavioral algorithms are challenging, even for such mundane actions as domestic water usage, and require good programming skills.

The results prove that $\mathrm{ABM}$ adds value to building system engineering by enabling experimentation with different hypotheses about occupant behavior in a relatively easy manner compared to other means (occupant surveys and fixture specific measurements). ABM enables studying of the building water system's performance before the actual infrastructure and the user is in place. This does not exclude the importance of acquiring measured data from households. It is crucial to validate the scenarios to the observed reality.

An ideal data set to validate the model would come from a case study building, from which one could simultaneously collect behavioral data through questionnaires and measurements. The problem of model calibration is the lack of reliable data. As Worthington \& Hoffman [26] note, the reality is that most research in water use behavior is based on a few unique data sets. Therefore the findings in literature are generally consistent. New and more exact data is needed to improve the calibration of the model. 


\section{Conclusions}

Model development, based on validation, enhancement and further development, and so on is a continuous process. The ABM model for domestic water use, which has been developed in the framework of the described research, could be further developed, although it has proved to be good enough to deliver the following insights about household water usage:

Different household types (households that have different demographic characteristics) have different water consumption patterns. For a busy single, most of the domestic water usage results from showering, whereas a retired couple uses substantial quantities of water for the toilet and the kitchen. Reducing water consumption of persons living alone and working outside home requires smart design of their bathing facilities.

There are interesting possibilities for model applications in participatory design, in the design of flexible building systems and in making cost effective design choices or policies. Water efficient buildings $v s$. water efficient home-technological innovations require systems thinking in design [32].

\section{Further Research}

For the future, there still remains a methodological question about the tools that should be developed for model human decision-making and behavior in buildings. While this paper presents one such implementation, it is not the only one, and the ideas presented here can be remixed for other implementations.

Modeling change in behavior ought to be a central focus of future model developments. This could take better advantage of the capabilities of ABMs.

It may be valuable to add some usability metrics to the model. The usability evaluation conducted in this research is limited compared to the one in Andrews et al. [19] and should be explored more as the building systems modeling continues. There is no optimal level of water usage to which the building engineers and occupants could refer in order to evaluate the efficiency and effectiveness of the building water system. Only in buildings that produce clean water (i.e., by harvesting rainwater) or recycle grey water (water that is not consumed in the toilet) does water quality, as an indicator of building water system performance, become important. Usability could be studied at the system level and the level of the fixture.

ABM could be a very useful tool to enable participatory design of building water systems, fixtures and appliances, in order to improve the design, and the same principles apply in model designs. A good model should be validated with its stakeholders, its clients. The stakeholders might have crucial knowledge of the system.

\section{Acknowledgments}

The authors would like to thank their Rutgers and TU Delft colleagues, for sharing their expertise and experience in building usability research and simulation modeling. Authors would like to especially mention Chris Davis from TU Delft and Handi Chandra Putra from Rutgers.

This research was in part supported by the National Science Foundation under Grant CMS-0725503 and the Civil engineering foundation of Finland (MVTT). 


\section{Conflict of Interest}

The authors declare no conflict of interest.

\section{References}

1. United States Environmental Protection Agency Water Supply in the U.S. Available online: http://www.epa.gov/WaterSense/pubs/supply.html (accessed on 2 May 2013).

2. CBS Statistics Netherlands Savings in Groundwater and Tap Water Use. Available online: http://www.cbs.nl/en-GB/menu/themas/natuur-milieu/publicaties/artikelen/archief/2011/2011 3349-wm.htm (accessed on 5 May 2013).

3. Shove, E. Converging conventions of comfort, cleanliness and convenience. J. Consum. Policy 2003, 26, 395-418.

4. Mayer, P.W.; DeOreo, W.B. Residential End Uses of Water; AWWA Research Foundation: Denver, CO, USA, 1999.

5. Heinrich, M. Water End Use and Efficiency Project (WEEP)_Final Report; No. 159; Branz: Jugendford, New Zealand, 2007.

6. Smith, A.; Ali, M. Understanding the impact of cultural and religious water use. Water Environ. J. 2006, 20, 203-209.

7. Gill, Z.M.; Tierney, M.J.; Pegg, I.M.; Allan, N. Low-energy dwellings: The contribution of behaviours to actual performance. Building Res. Inform. 2010, 38, 491-508.

8. Krantz, H. Matter that Matters: A Study of Household Routines in a Process of Changing Water and Sanitation Arrangements. PhD Thesis, Linköping University, Linköping, Sweden, 2005.

9. Andrews, C.J. Building a micro foundation for industrial ecology. J. Ind. Ecol. 2000, 4, 35-51.

10. Schuetze, T. Dezentrale Wassersysteme im Wohnungsbau internationaler Großstädte am Beispiel der Städte Hamburg in Deutschland und Seoul in Süd-Korea (Decentralized Water Systems in Housing Estates of International Big Cities Using the Example of Hamburg in Germany and Seoul in South Korea); Books on Demand: Norderstedt, Germany, 2005; p. 496.

11. Deoreo, W.D.; Mayer, P.W.; Dietemann, A.; Skeel, T.; Lewis, D.M.; Smith, J. Retrofit realities. Am. Water Works Assoc. J. 2001, 93, 58-72.

12. Jorgensen, B.; Graymore, M.; O’Toole, K. Household water use behavior: An integrated model. J. Environ. Manag. 2009, 91, 227-236.

13. Environmental Management Center (EMC). Wise Water-A Microsoft Excel ${ }^{T M}$ Based Spreadsheet $^{2}$ Application; United Nations Environment Program (UNEP): Mumbai, India, 2008.

14. Schuetze, T.; Tjallingi, S.P.; Correlje, A.; Ryu, M.; Graaf, R.; van der Ven, F. Every Drop Counts: Environmentally Sound Technologies for Urban and Domestic Water Use Efficiency, 1st ed.; Schuetze, T., Tjallingi, S.P., Eds.; UNEP: Nairobi, Kenia, 2008; p. 197.

15. Nikolić, I. Co-Evolutionary Method for Modelling Large Scale Socio-Technical Systems Evolution. Ph.D. Thesis, Technische Universiteit Delft, Delft, The Netherlands, 2010.

16. Castiglione, F. Agent based modeling. Scholarpedia 2006, 1, doi:10.4249/scholarpedia.1562.

17. Moglia, M.; Perez, P.; Burn, S. Modelling an urban water system on the edge of chaos. Environ. Modell. Softw. 2010, 25, 1528-1538. 
18. Janssen, M.; Jager, W. An integrated approach to simulating behavioural processes: A case study of the lock-in of consumption patterns. J. Artif. Soc. Soc. Simul. 1999, 2, 21-35.

19. Andrews, C.J.; Yi, D.; Krogmann, U.; Senick, J.A.; Wener, R.E. Designing buildings for real occupants: An agent-based approach. IEEE. Trans. Syst. Man. Cybern. A 2011, 41, 1077-1091.

20. Ajzen, I. The theory of planned behavior. Organ. Behav. Hum. Decision. Proc. 1991, 50, 179-221.

21. Robbins, R.W.; Wallace, W.A. Decision support for ethical problem solving: A multi-agent approach. Decis. Support. Syst. 2007, 43, 1571-1587.

22. Foekema, H.; van Thiel, L.; Lettinga, B. Watergebruik Thuis 2007 (Water use at home 2007); TNS NIPO: Amsterdam, The Netherlands, 2008.

23. Wilensky, U. NetLogo Home Page. Available online: http://ccl.northwestern.edu/netlogo/ (accessed on 5 May 2013).

24. Wilkes, C.R.; Mason, A.D.; Hern, S.C. Probability distributions for showering and bathing water-use behavior for various U.S. subpopulations. Risk. Anal. 2005, 25, 317-337.

25. Schwartz, S.H. Universals in the content and structure of values: Theoretical advances and empirical test in 20 countries. Advan. Exp. Soc. Psychol. 1992, 25, 1-66.

26. Worthington, A.C.; Hoffman, M. An empirical survey of residential water demand modelling. J. Econ. Surv. 2008, 22, 842-871.

27. Ferrara, I. Household Behaviour and the Environment, Reviewing the Evidence; Organization for Economic Cooperation and Development: Paris, France, 2008; pp. 153-180.

28. U.S. Energy Information Association. Cooking Trends in the United States: Are We Really Becoming a Fast Food Country? Available online: http://www.eia.doe.gov/emeu/recs/ cookingtrends/cooking.html (accessed on 7 July 2013).

29. Kant, A.K.; Graubard, B.I. Eating out in America, 1987-2000: Trends and nutritional correlates. Prev. Med. 2004, 38, 243-249.

30. Corraliza, J.A.; Berenguer, J. Environmental values, beliefs, and actions: A situational approach. Environ. Behav. 2000, 32, 832-848.

31. Blokker, E.J.M.; Vreeburg, J.H.G.; van Dijk, J.C. Simulating residential water demand with a stochastic end-use model. J. Water Resour. Plan. Manag. 2010, 136, 19-26.

32. Scott, K.; Quist, J.; Bakker, C. Co-Design, Social Practices and Sustainable Innovation: Involving Users in a Living Lab Exploratory Study on Bathing. In Proceedings of Paper for the "Joint Actions on Climate Change" Conference, Aalborg, Denmark, 8-9 June 2009.

(C) 2013 by the authors; licensee MDPI, Basel, Switzerland. This article is an open access article distributed under the terms and conditions of the Creative Commons Attribution license (http://creativecommons.org/licenses/by/3.0/). 